\title{
Der Wandel von Parteien als Interessenvertretungen
}

\section{Lawson, Kay und Thomas Poguntke (Hrsg.): How Political Parties Respond. Interest Aggregation} Revisited, Routledge, London 2004, 271 Seiten, € 106,50.

Politische Parteien unterliegen permanenten Wandlungsprozessen, denn sie müssen sich an Veränderungen der sie umgebenden Umwelten anpassen und mit ihrer Programmatik, ihrer Organisation und ihren Kommunikationsstrategien neuen Herausforderungen Rechnung tragen. Damit sie selbst nicht nur Getriebene des Prozesses sind, entwickeln sie eigene Strategien, Handlungsformen und Instrumente des Umgangs mit den Veränderungen.

Da die Befassung mit politischen Parteien zum Kernbestand der politikwissenschaftlichen Forschung gehört, hat sich diese umfassend mit Parteienwandel in seinen verschiedenen Facetten auseinandergesetzt. Innerhalb dieser Literatur versucht der von Kay Lawson und Thomas Poguntke herausgegebene Sammelband einen eigenen Schwerpunkt zu bilden, indem er die Funktionen der Interessenaggregation und der Responsivität politischer Parteien in den Vordergrund stellt und der Frage nachgeht, ob und wie Parteien diese Funktionen unter den sich wandelnden Bedingungen noch wahrnehmen können. Der Band zielt auf die gesellschaftliche Verankerung von Parteien und deren Aufgaben im Prozess der politischen Interessenvermittlung sowie als Vermittler demokratischer Legitimation ab - das Themenfeld, auf dem beide Herausgeber langjährige Expertise aufweisen. Wie Lawson im Fazit konstatiert, ist es mit der normativ zu verstehenden Wahrnehmung der Funktion der Interessenaggregation von Parteien empirisch nicht zum Besten bestellt. Die häufig beklagten Integrations- und Repräsentationsdefizite werden auch von den Autoren der einzelnen Beiträge konstatiert, ohne jedoch glücklicherweise in ein allgemeines Lamento zu verfallen. Wie ein roter Faden zieht sich durch das Buch, dass die Parteien sich professionalisiert, medialisiert und etatisiert haben - ein Befund, der für die deutschen Großparteien auch schon getroffen wurde. Die Stimmengewinnlogik hat längst klar die Überhand gegenüber der reinen Wahrnehmung beziehungsweise Kanalisierung der Mitglieder- und Wählerpräferenzen und ihrer Umsetzung in programmatische Leitlinien gewonnen.

Von allen etablierten europäischen Großparteien hat die britische Labour Party wohl den umfassendsten Wandel ihrer Organisation, ihrer Programmatik und ihres Kommunikationsmanagements in der nun zu Ende gehenden Ära ihres Parteivorsitzenden Tony Blair vollzogen. Dieser Wandel von der noch zu Beginn der 1980er Jahre programmatisch traditionellen, von den Gewerkschaften dominierten Linkspartei hin zur modernisierten Sozialdemokratie, die gekonnt auf die wahlentscheidenden Mittelschichten abzielt und moderne Kommunikationsinstrumente lange Zeit nahezu perfekt für sich zu nutzen wusste, wird von James E. Coronin sowie von Patrick Seyd und Paul Whiteley in zwei Beiträgen mit verschiedenen Schwerpunkten analysiert. Während Coronin „New Labour“ unter historischer Perspektive als notwendige Abkehr von „Old Labour“ in Folge massiver gesellschaftlicher Veränderungen und der Erfolge der Politik des „Thatcherismus“ betrachtet, untersuchen Seyd und Whiteley die Regierungspolitik der Blair-Administration mit Blick auf Verluste bei Mitgliedern und der einstigen Stammwählerschaft, die sich von der Partei nicht mehr repräsentiert fühlen, was die Autoren als problematischen Aspekt der Veränderungen hervorheben.

Den von der Labour Party mit Vehemenz verfolgten Kurs hin zur politischen Mitte, gemeint als programmatische Annäherung an liberale und konservative Mitbewerber im Par- 
teiensystem, konstatiert Nicholas Aylott auch für die skandinavische Sozialdemokratie, wenn auch in gemäßigterer Form. Dieser geht einher mit einer loser werdenden Bindung zu den Gewerkschaften, ohne dass diese aber bedeutungslos geworden ist, vielmehr insbesondere auf lokaler Ebene noch erhebliche Relevanz hat. Eine Schwächung des Mitgliedereinflusses, Professionalisierung und Modernisierung des Kommunikationsmanagements zählen für Aylott zu den weiteren Veränderungsmerkmalen der drei sozialdemokratischen Parteien in Dänemark, Schweden und Norwegen. Unterstützung erhält er von Karina Pedersen, die für dänische Parteien insgesamt zu ähnlichen Ergebnissen kommt, gleichzeitig jedoch betont, dass trotz abnehmender Relevanz der Mitglieder für das Organisationsleben der Parteien diese weiterhin eine nicht zu vernachlässigende Größe darstellen. Damit verweist Pedersen auf einen wichtigen Aspekt, den manche Kritiker der Literatur zum Parteienwandel übersehen oder zu oberflächlich hineinlesen wollen. Allgemein wird darin von einem Bedeutungsrückgang der Parteimitglieder gesprochen, jedoch nicht von einem weitgehenden oder gar vollständigen Bedeutungsverlust.

Die relative Geringschätzung der Mitgliedschaft und die eindeutige Orientierung auf die Stimmengewinnlogik und damit einhergehend auf moderne Formen der politischen Kommunikation, insbesondere in Wahlkämpfen, heben Luis Ramiro und Laura Morales in ihrer lesenswerten Fallstudie zu den spanischen Großparteien PSOE und PP hervor. Die spanischen Parteien sind aufgrund der späten Demokratisierung nicht aus Vorfeldorganisationen hervorgegangen, noch haben sie enge Bindungen zu ihnen aufgebaut und sind auch nur gering in einzelnen sozialen Gruppen verankert. Sie betreiben daher kaum Interessenaggregation, sondern verfolgen laut Ramiro und Morales den Marketing-Ansatz der Politik mit der Anpassung von Wahlprogrammen an Wählerpräferenzen.

Dass man diesen Ansatz aber sogar noch deutlich weiter treiben und nahezu vollständig losgelöst von Mitgliedereinflüssen und gesellschaftlichen Strömungen implementieren kann, zeigt Suzanna Pshizova in ihrer überzeugenden Analyse der russischen Parteien auf. In diesen dominieren Führungspersönlichkeiten, deren Images von Beratern kreiert, massenmedial transportiert und nach modernen Marketing-Gesichtspunkten evaluiert werden: „Most look like a one-man band with an attractive advertising sign and actively practise professional marketing techniques." (S. 246) Parteiorganisation und Programme spielen mit Ausnahme der Kommunisten keine nennenswerte Rolle. Pshizova stellt selbst schon den (naheliegenden) Bezug zur Forza Italia Silvio Berlusconis her, die Jonathan Hopkin in seinem Beitrag als „business firm party“ (S. 189) bezeichnet. Eine solche wird nach Hopkin von einem privaten Unternehmen gegründet, welches die Partei kontrolliert und seine Interessen in der Politik verfolgt. Mitglieder spielen keine Rolle, kapitalintensive Wahlwerbung in den Massenmedien bestimmt die Außendarstellung der Partei, die im Falle der Forza Italia ganz auf ihren Gründer und Vorsitzenden Berlusconi zugeschnitten ist und von diesem eindeutig dominiert wird. Dass Parteiführungen das innerparteiliche Leben zu großen Teilen auch in etablierten Parteien wesentlich prägen, zeigt Nicolas Sauger in seiner Fallstudie zur französischen UDF mit Blick auf deren Programmentscheidungen auf.

Die einzelnen Kapitel verdeutlichen in unterschiedlichen Perspektiven und Aspekten anschaulich und analytisch fundiert den Wandlungsprozess der europäischen Parteien vom Modell der „catch-all party“ zu professionalisierten Formen der Medienkommunikation. Sie haben sich zu stärker führungszentrierten Organisationen entwickelt, deren Mitglieder an Bedeutung einbüßen, deren Programmatik sich in erheblichem Ausmaß an den Vorstellungen der Wähler orientiert und die zur Deckung der kapitalintensiven Außendarstellung 
auf staatliche Mittel zurückgreifen (wobei letzteres von den im Band analysierten nur bei den skandinavischen Parteien im Vordergrund steht).

Den Herausgebern ist es gelungen, mit dieser Zusammenstellung vielfältige Gesichtspunkte des Parteienwandels zu beleuchten. Ihre Einleitung und ihr Schlusskapitel stellen die einzelnen Beiträge in einen Gesamtkontext. Der Vorzug der Vielschichtigkeit hat allerdings einen Nachteil: Die Heterogenität lässt keine einheitliche Sichtweise zu. Einzelne Beiträge, wie etwa der zur Verteilungspolitik in Berlin (Louise K. Davidson-Smith) passen allenfalls sehr eingeschränkt in das Analyseraster und den Gesamtrahmen dieses ansonsten beachtenswerten Sammelbandes. Für Parteienforscher, aber auch für den an Wandel von Parteien allgemeiner interessierten Leser bietet die Publikation eine Reihe von lesenswerten Informationen und gut begründeten Schlussfolgerungen.

Uwe Jun

\section{Internationale Wahlbeobachtung als Demokratisierungshilfe}

Münzing, Ekkehard: Internationale Wahlbeobachtung nach dem Ende des Ost-West-Konflikts. Unter besonderer Berücksichtigung der Afrikanischen Union, des Carter Centers, des Commonwealth of Nations, der Organisation Amerikanischer Staaten und der Organisation für Sicherheit und Zusammenarbeit in Europa, Poli-c-books, Berlin 2005, 328 Seiten, € 34,80.

Gut 15 Jahre nach Ende des Ost-West-Konflikts und dem damit einhergehenden Demokratisierungsschub ist die Legitimität von Wahlen beziehungsweise Wahlprozessen immer noch zentrales Thema der Politik. Allein für das Jahr 2006 sei hier auf die Präsidentschaftswahl in Weißrussland und die Wahl im Kongo - und deren Absicherung durch einen EUMilitäreinsatz unter Beteiligung deutscher Soldaten - hingewiesen. In solchen Situationen rücken die Berichte der Wahlbeobachter in den Fokus der internationalen Öffentlichkeit. Sie gelten als ein Instrument der Demokratisierungshilfe und sind inzwischen so häufig eingesetzt worden, dass mitunter von einer „booming industry“ und „monitoring industry“ (S. 4) gesprochen worden ist.

Die Wahlbeobachtung und die internationalen Institutionen, die sie durchführen, werden in der hier anzuzeigenden lesenswerten Dissertation mit hohem aktuellen politischen Bezug von Ekkehard Münzing einer vergleichenden Analyse und Bewertung unterzogen. Im ersten Teil werden Grundlagen und Hintergründe der Wahlbeobachtung analysiert und eine gründliche theoretische Rechtfertigung für das große Engagement der zahlreichen Akteure geliefert. Aufbauend auf der Kantschen These des demokratischen Friedens wird überzeugend begründet, dass die „Etablierung und Konsolidierung demokratischer Systeme in bisher undemokratischen Staaten im originären Eigeninteresse der demokratischen Staaten “ (S. 18) liegt. Schließlich wird das große Spektrum von Akteuren und Instrumenten der Demokratisierungshilfe herausgearbeitet, bei denen internationale Wahlbeobachtungen nur ein Instrument unter vielen sind.

Münzings Analyse der internationalen und regionalen Menschenrechtsverträge ergibt ein disparates Ergebnis. So hat zwar „eine breite völkerrechtliche Verankerung des Rechts auf 